\title{
Research Paper \\ The Effectiveness of Schema Therapy Techniques in Mental Health and Quality of Life of Women With Premenstrual Dysphoric Disorder
}

\author{
*Sara Mohtadi Jafari ${ }^{1}$, Hassan Ashayeri $^{2}$, Parinaz Banisi ${ }^{3}$ \\ 1. MA in Public Psychology, Department of General Psychology, Faculty of Humanities, Tehran West Branch, Islamic Azad University, Tehran, Iran \\ 2. Professer in Basic Sciences, Faculty of Basic Sciences, Iran University of Medical Sciences, Tehran, Iran. \\ 3. PhD. in Psychology, Department of General Psychology, Faculty of Humanities, Tehran West Branch, Islamic Azad University, Tehran, Iran.
}

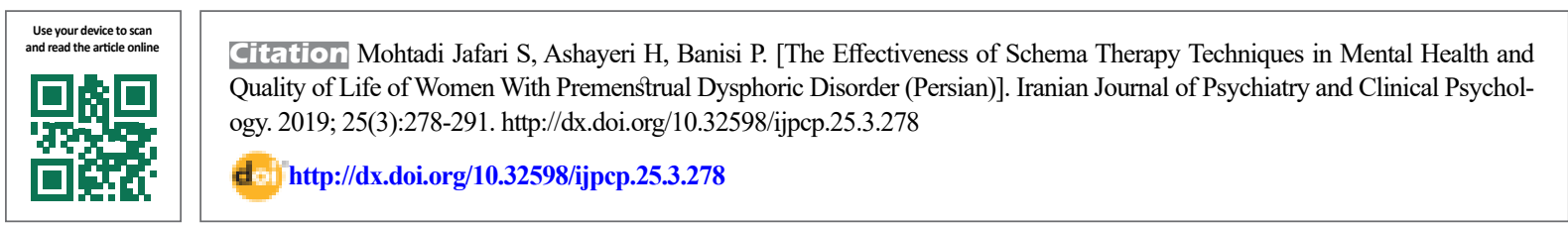

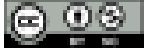

Received: 15 Dec 2018 Accepted: 09 Jun 2019 Available Online: 01 Oct 2019

Key words: Premenstrual dysphoric disorder, Schema therapy, Mental health, Quality of life

\section{A B STRACT}

Objectives The present research investigated the effect of schema therapy techniques on the mental health and quality of life in women with premenstrual dysphoric disorder.

Methods This quasi-experimental study was conducted with a pre-test, post-test design. Moreover, 36 women were randomly assigned into two groups and received ten training sessions. Research instruments were Mental Health and Quality of Life (to measure the effectiveness of training) Questionnaires. Results There was a significant difference between the two groups in the mental health scores in the components of depression, social function disorder, and anxiety. Moreover, regarding the quality of life test scores, there were significant differences in mental health and physical health components between the study groups. In the follow-up phase, the same results in mental health and quality of life also affected the health environment.

Conclusion The training of this method can affect the intended components.

\section{Extended Abstract \\ 1. Introduction

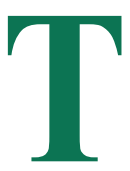 \\ The current research explored the effec- tiveness of training schema therapy tech- niques on general health and quality of life of women with the premenstrual dysphoric disorder.}

A semi-experimental design with a pre-test, post-test, and a control group was used. At first, the central area of Tehran City, Iran, was selected among 5 parts of it (north, south, east, west, and center); then, district 10 was randomly se- lected from 4 districts in the central part of Tehran, including districts $9,10,11$, and 12 . The statistical population of this study included all women referring to cultural centers and community centers of district 10 of Tehran municipality, during the first three months of 2018. Premenstrual Dysphoric Screening test and Young Maladaptive Schemas test with 90 items were conducted.

According to the scores obtained from these tests initially 48 people were considered suitable for the study (in the first test, persons who scored higher than the cutoff point of 28 , and in the second one, persons who received a score higher than 5 in the category of questions related to each schema), -

* Corresponding Author:

Sara Mohtadi Jafari, MA

Address: Department of General Psychology, Faculty of Humanities, Tehran West Branch, Islamic Azad University, Tehran, Iran. Tel: +98 (912) 2583087

E-mail: saramohtadijafari@gmail.com 
Finally, 36 persons were selected by purposive sampling method from those who had the disorder.

Then, they were randomly divided into the test and control groups (18 persons per group). In each group, three more persons were selected to prevent sample drop and the threat of non-scientific research. To collect data, Goldberg General Health Test (28 items) was used. This test measures 4 subscales of signs of individual's anxiety and insomnia, depression, physical health, and social relations during the last month. The lower scores indicate better mental health.

Moreover, the Quality of Life Scale of World Health Organization (WHO) (28 items) was used to measure 4subscales of general mental health, physical health, social functions, and environmental health. The higher scores indicate higher quality of life. These two tests were used to study the effectiveness of training schema therapy. The test group received training for 10 two-hour sessions (105 minutes training and 15 minutes break and catering) every week (one session per week), for 2. 5 months (from July to September 2018). The control group received no training during this period.

The training was conducted in group and by the researcher, based on Dr. Fata and Dr. Motabi's therapeutic package. Stages of the schema therapy are in two dimensions of measuring and training, and changing schemas. Four techniques were used to change schemas, as follows: cognitive techniques, in which the aim of change is the central belief (including identifying the central belief, identifying keywords of the belief and their definitions, the percent of believing them, finding advantages and disadvantages, evidence confirming or rejecting the central belief, and writing training cards).

The aim of cognitive techniques is creating a healthy sound in mind against schemas' sounds. Experimental techniques focus on memories, mental images, body feelings, and emotions (including imagination, imaginary conversation, blank seat technique, writing a letter to the cause of the memory, and finally reading the letter and evacuation of protective anger). Behavioral pattern-breaking focuses on inefficient coping styles (avoidance, extreme compensation, and submission, including increased motivation for change, practicing healthy behaviors through imagination and role-playing, teaching to overcome barriers to behavior changes, making important changes in life, and finally stopping treatment, if it is impossible to break behavior pattern, for 2 to 3 months).

Relationship therapy is effective in meeting five emotional needs (safety, autonomy, self-expression, fun and playfulness, and realistic restrictions). Here, relationship therapy means relations with the group's teacher and other members. Finally, to ensure the survival of the training effect, a follow-up test was conducted in the test group after one month. The obtained results were analyzed using SPSS and descriptive statistics (mean and standard deviation) and inferential statistics (multivariate covariance analysis and t-test).

\section{Conclusion}

Training the schema therapy techniques made a significant difference in three scales of 4 measured items of mental health test, including symptoms of depression, anxiety and insomnia, and social dysfunction. Those also made significant changes in two subscales of 4 measured items of quality of life, including mental health and physical health between the two groups.

At one-month follow-up, it was found that in terms of mental health, the impact of training the schema therapy techniques on this statistical society is permanent; in terms of quality of life, training the schema therapy techniques is effective in enhancing the health of the environment of women with the premenstrual dysphoric disorder.

According to the Diagnostic and Statistical Manual of Mental Disorders, 5th Edition (DSM-5) criteria for premenstrual dysphoric disorder, there are one or more of the following symptoms in each cycle: 1 . Tangible emotional instability such as mood fluctuations, sadness or crying, more sensitivity to rejection; 2. Irritability or tangible anger and increased interpersonal conflicts; 3 . Tangible depression, frustration, and self-condemnation; 4. Anxiety, tangible tension, or feeling excitement. According to the applied questionnaire, changing these items reduces mental health in women with this disorder.

According to these criteria, symptoms of this disorder (including: 1. less interest in performing routine activities (e. g. job, education, friends, and entertainment); 2. mental problem in focus; 3 . indolence, fatigue, tangible energy loss; 4. intangible appetite loss, overeating, or eager to eat a certain food; 5. oversleeping or insomnia, 6. feeling of power failure); indicate reduced quality of life of women with this disorder. In this research, it was explained to the test group that in each menstrual cycle, people with premenstrual dysphoric disorder feel more stress, anxiety, and depression.

Thus, their social function and biopsychological health will be reduced. Particularly when they have inconsistent schemas, these moods are exacerbated. Training the sche- 
ma therapy techniques helps people to less experience these hard conditions during disorder cycle; therefore, this disorder has a less destructive effect on their mental health and quality of life.

\section{Ethical Considerations}

\section{Compliance with ethical guidelines}

All ethical principles were observed in this study. A written informed consent was obtained from the participants and they were assured of the confidentiality of their information.

\section{Funding}

This paper was extracted from a master thesis approved by the Department of General Psychology, Faculty of Humanities at Islamic Azad University of Tehran West Branch. We received no any specific grant from funding agencies in the public, commercial, or not-for-profit sectors.

\section{Authors contributions}

The authors had same contribution in preparing this paper.

\section{Conflicts of interest}

The authors declared no conflicts of interest. 


\title{
اثربخشى آموزش فنون طرحواره درمانى بر سلامت روان و كيفيت زندتى زنان مبتلا به اختلال ملال

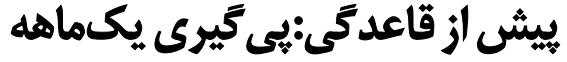

\author{
"سارا مهتدى جعفرى' ، حسن عشايرى '، بريناز بنىسى' \\ ا. كارشناس ارشد روانشناسى عمومى، كروه روانشناسى عمومى، دانشكده علوم انسانى، واحد تهران غرب، دانشكاه آزاد اسلامى، تهران، ايران.

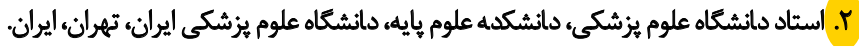

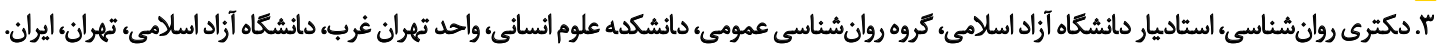

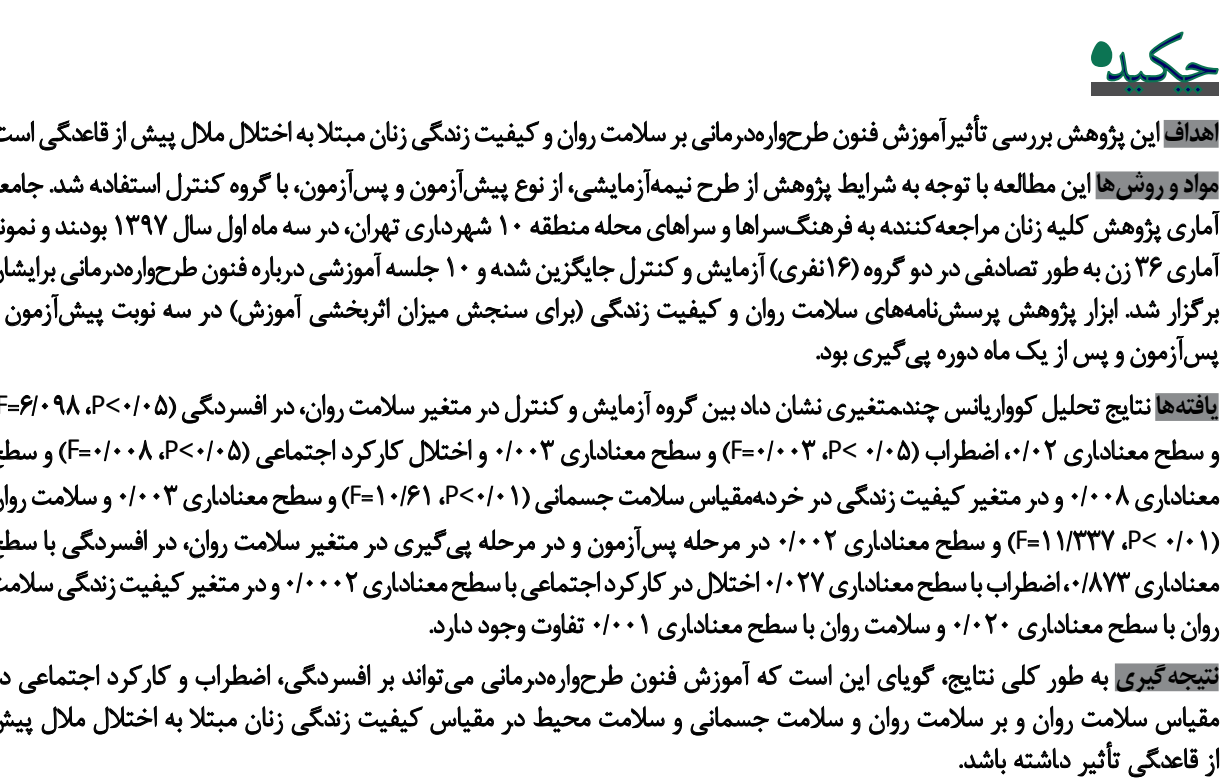

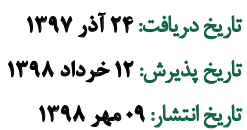

كليدوازوها:

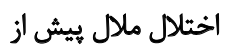

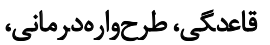
سلامت روان،كيفيت زنديكى قراعي

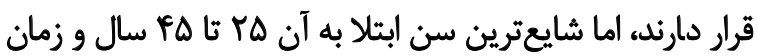

daleo

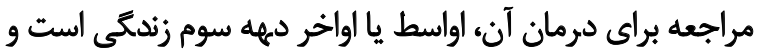

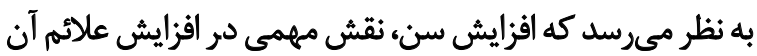

داشته باشد [ب].

بنا به تعريف سازمان بهداشت روانى، سلامت روانى حالتى از

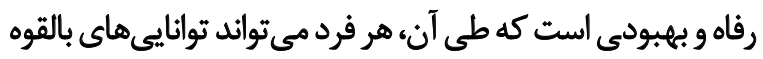

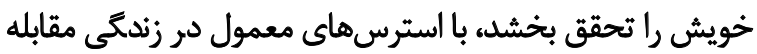

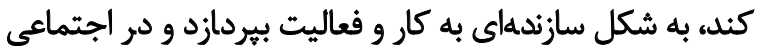

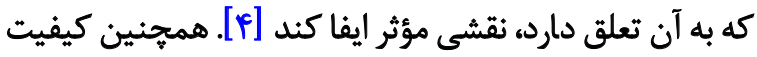

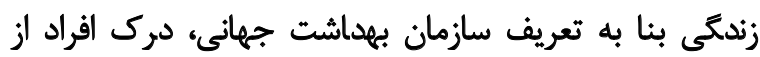

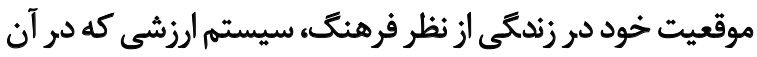

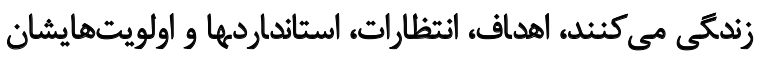

در زيستشناسى انسان خصوصأ دئياى آكنده از تجارب و فراز

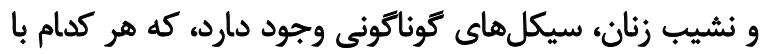

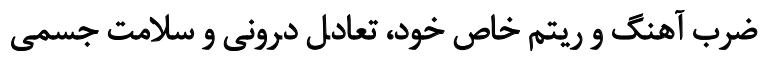

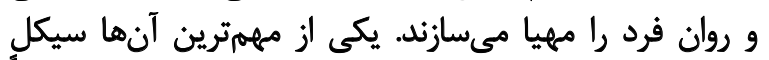

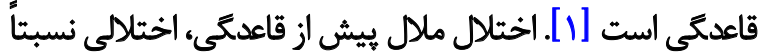

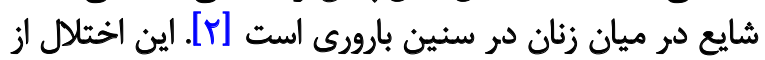

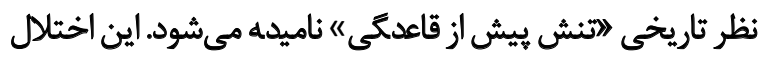

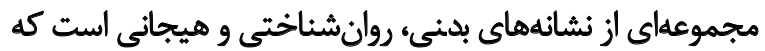

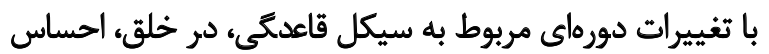

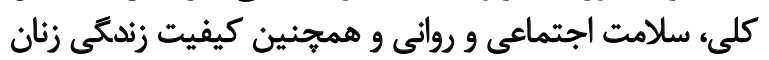

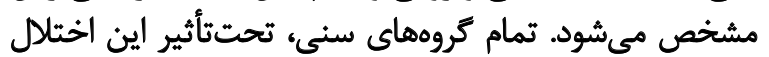


نسبت به طرد؛ r. تحريكيذيرى يا خشمه محسوس يا افرايش

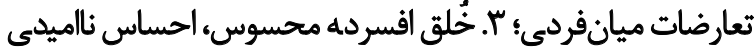

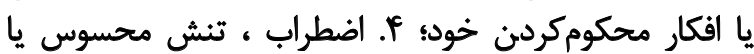

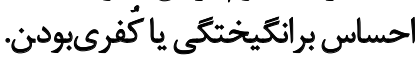
تغيير اين موارد طبق زيرمقياسهاى يرسش إنامه، سبب كاهش

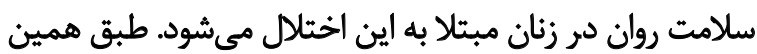

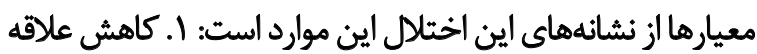

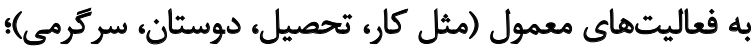

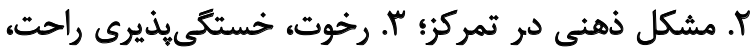

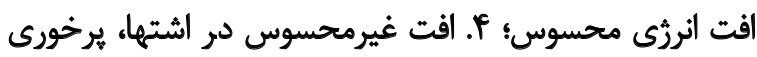

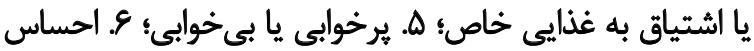

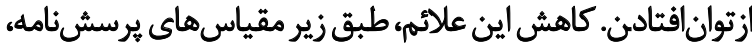

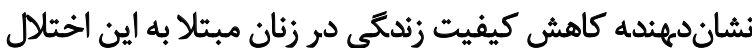

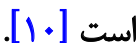

بيش از اين هيج إثوهشى مبتنى بر اثربخشى فنون

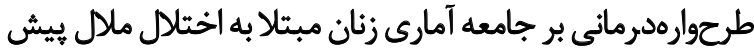

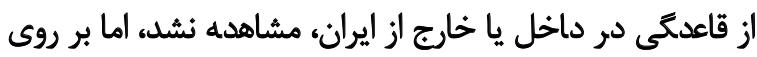

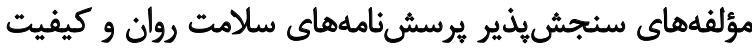

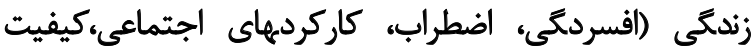

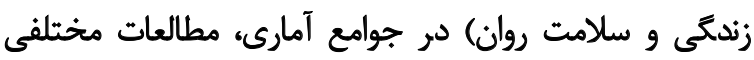

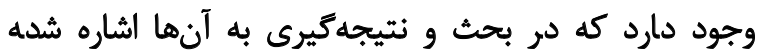

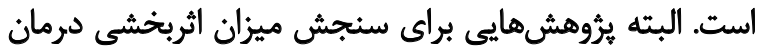

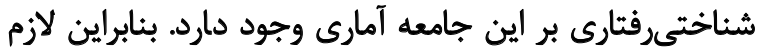

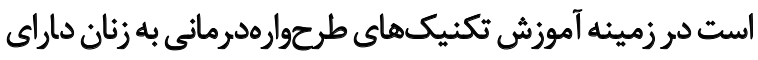

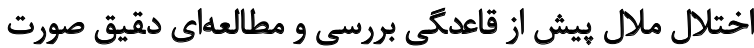

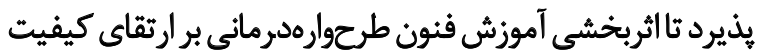

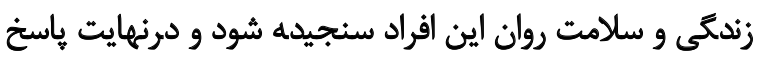

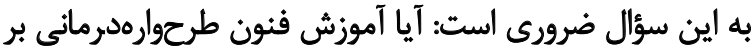

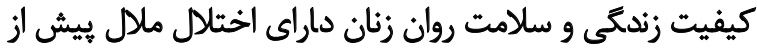

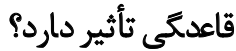

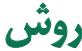

در يرُوهش حاضر آزمودنىها از طريق نمونهكيرى هدفميند

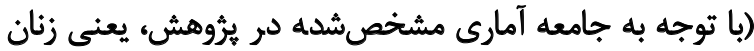

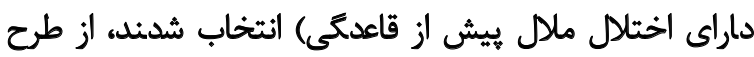

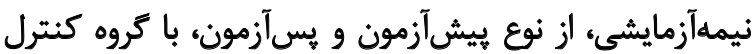

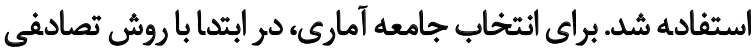

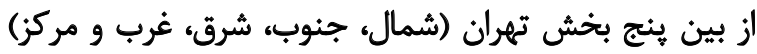

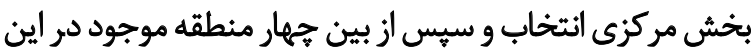

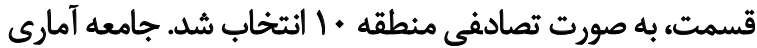

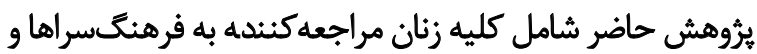

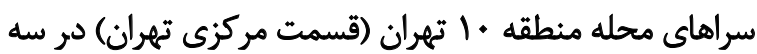

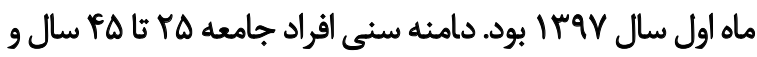

است. بـ كيفيت زندئى موضوعى كاملاً ذهنى است و ديكران

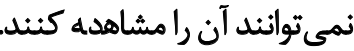

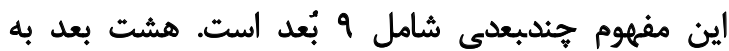

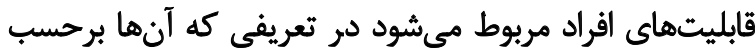

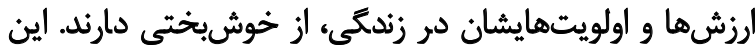

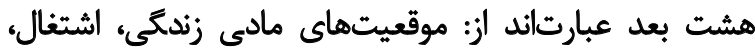

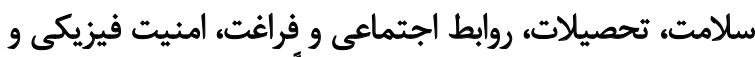
اقتصادى، دولت و حقوق اساسى و نهايتأ محيط زندئى ونى و طبيعى

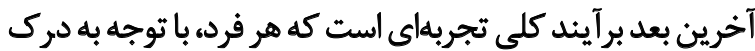
شخصى از كيفيت زندگَى دارد. [ه]]

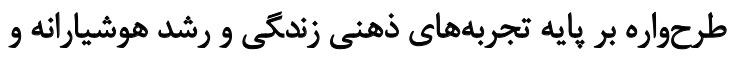

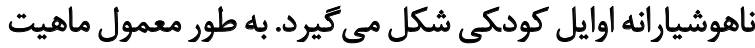

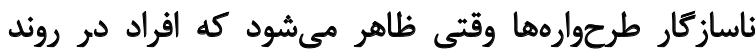

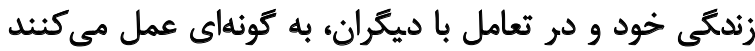

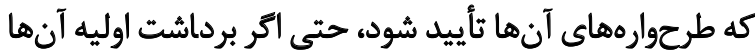

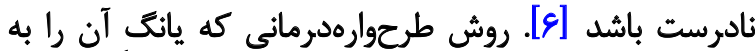

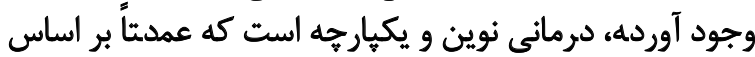

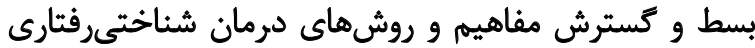

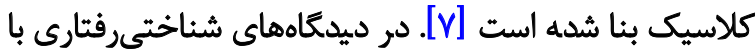

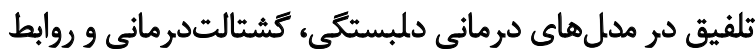

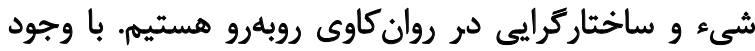

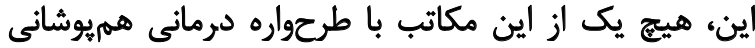

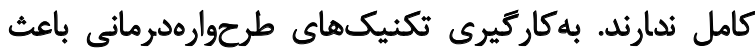

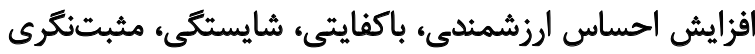
و اعتمادبهنفس مىشود [1]].

طرحوارهدرمانى در دو مرحله سنجش و آموزش، همجينين

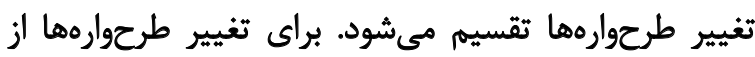

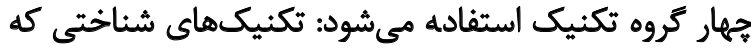

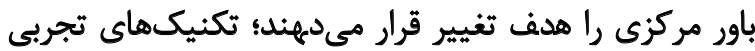

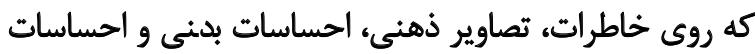

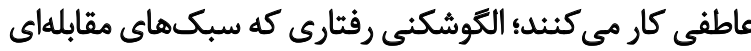

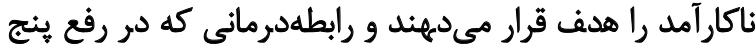

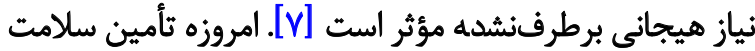

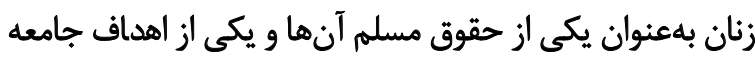

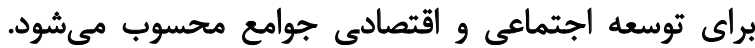

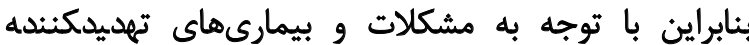

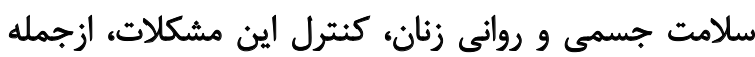
اولويتهاي بهداشتى است روانى زنان،

طبق معيارهاي DSM-5 براي اختلال ملال بيش از قاعدمَى ئي

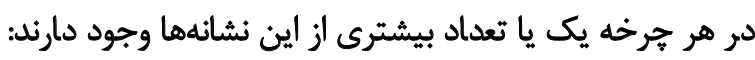

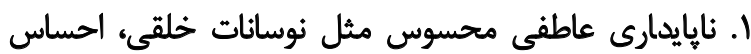

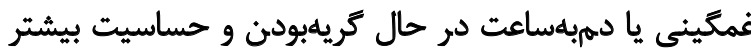


كروه آزمايش در يك جلسه توجيهي، يس ازئه يركردن

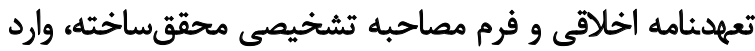

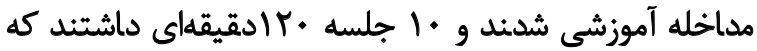

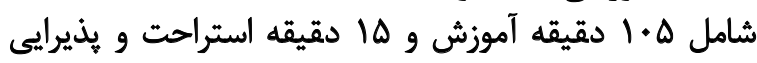

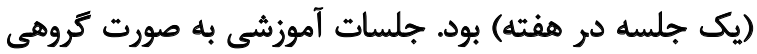

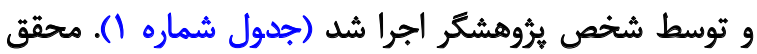

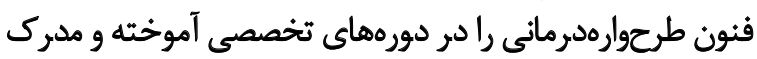

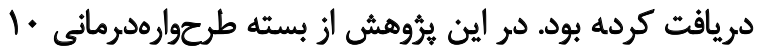

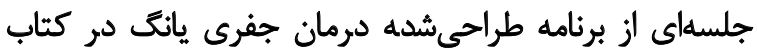

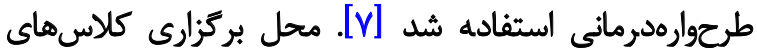

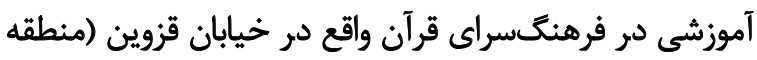

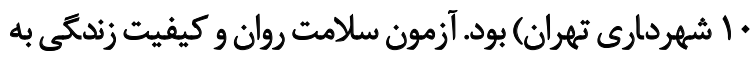

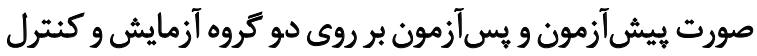

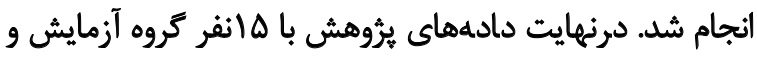

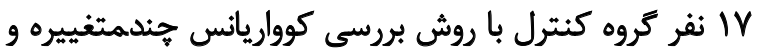

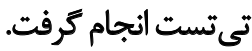

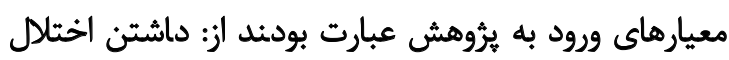

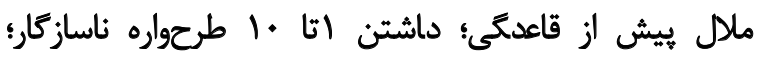

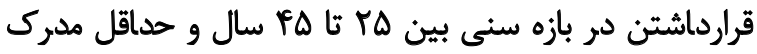

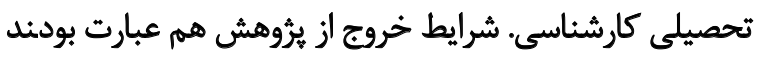

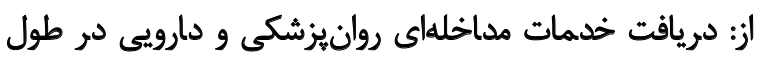

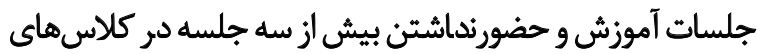

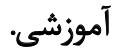

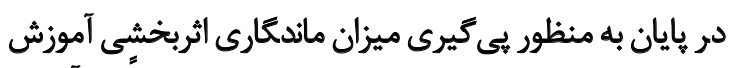

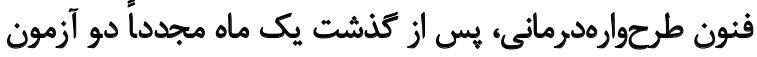

حاقل سطح تحصيلات كارشناسي، بدون درنظركرفتن وضعيت

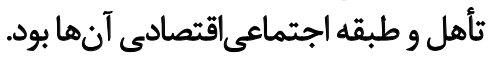

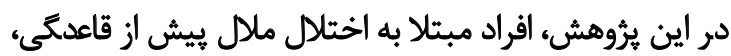

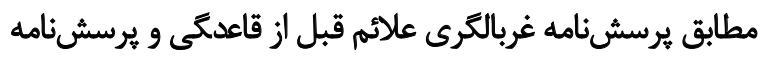

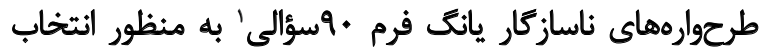

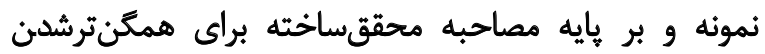

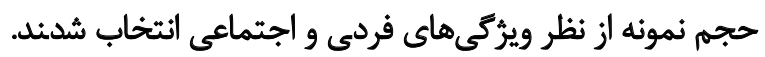

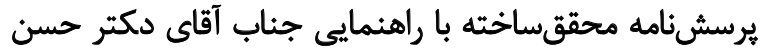

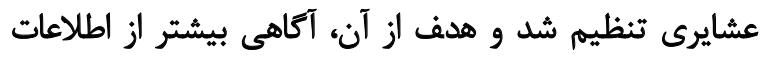

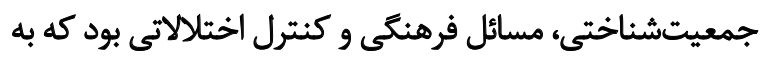

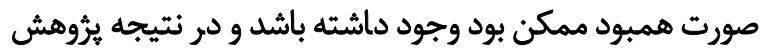

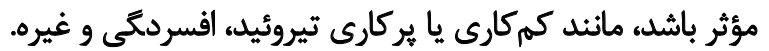

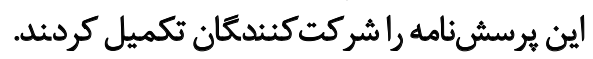

نخست از بين هاب هيرسشنامه غربالكَى علائم قبل از

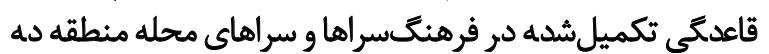

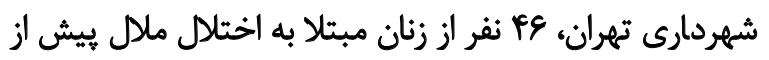

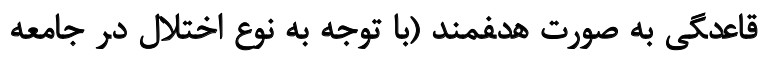

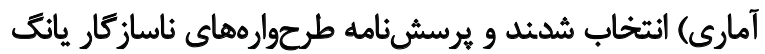

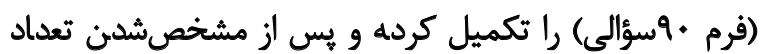

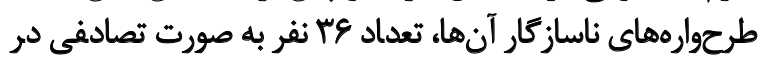

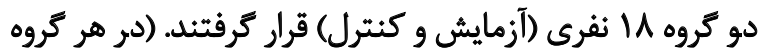

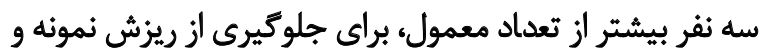

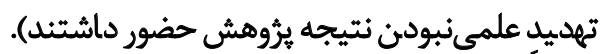

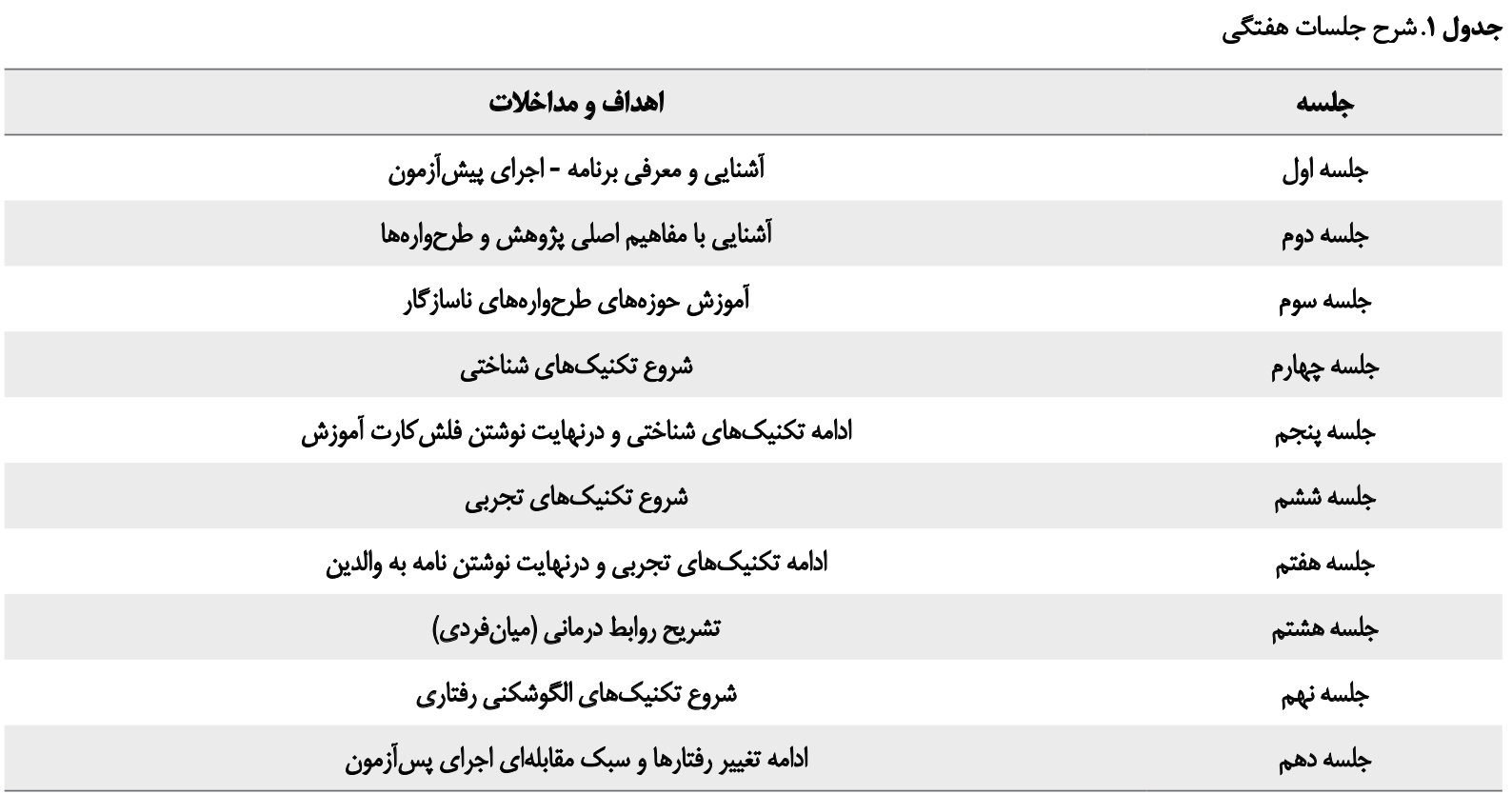

مجله روانيزنشى وروانشناسى بالينى ايران 


$$
\text { برسش نامه سامت روان تخلدبرتى }
$$

اين يرسشنامه آزمونى است با ماهيت جندئانه وخوداجراكه

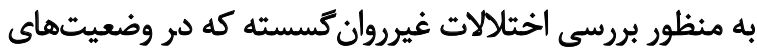

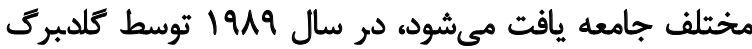

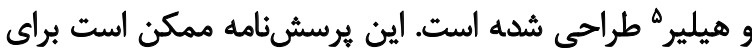

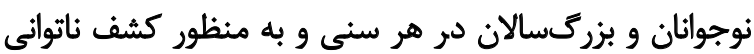

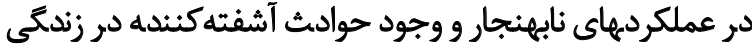

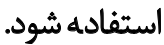

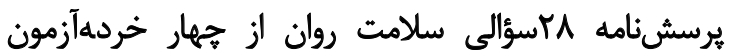

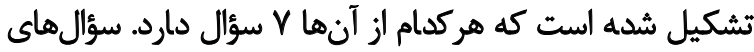

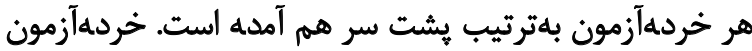

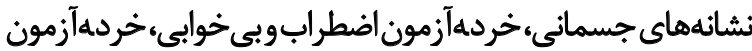

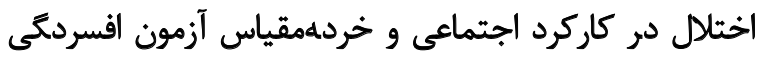

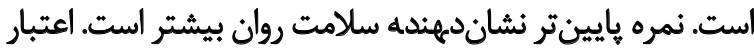

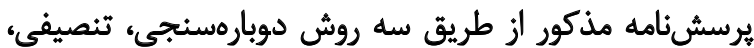

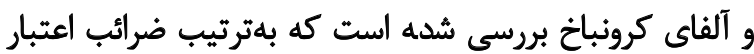

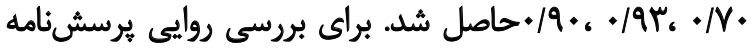

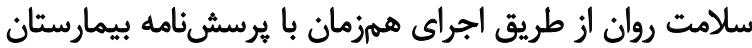

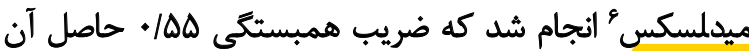

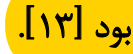

\section{يرسش نامه كيقيت زئدكّى سازٔمان بهداشت جهاني v}

اين : يرسشنامه شامل \&Y يرسش است كه در سال 1999،

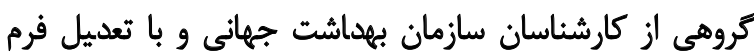

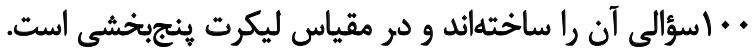

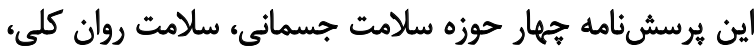

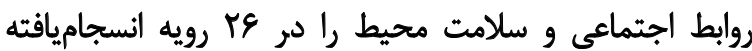

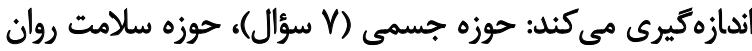

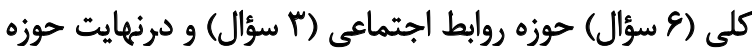
سلاهت هحيط (1 سؤال).

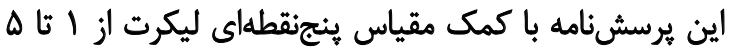

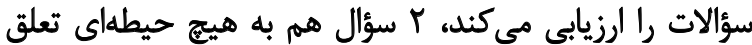

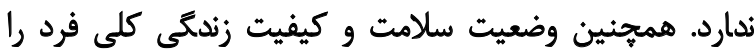

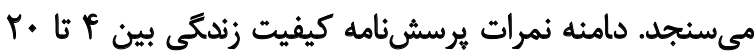

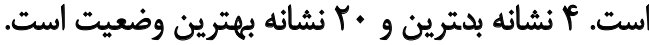

اسكوبنكتون و همكاران در گزارشي از طرف كروه كيفيت

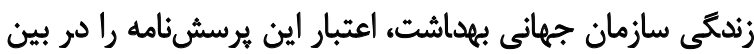

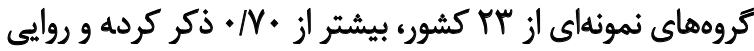

4. Genereal Health Questtionnaire (GHQ-28)

5. Goldberg \& Hiler

6. Middlesex Hospital

7. WHOQOL-BREF-28

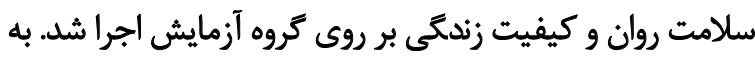

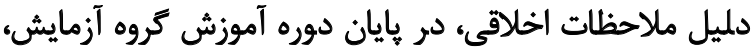
اين دوره • اجلسهاى براى كروه كنترل نيز بركزار شد.

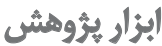

\section{يرسش يامه غربالكرى علاثم ملال بيش از قاعدكى"}

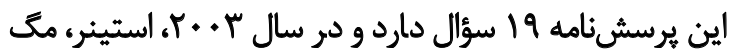

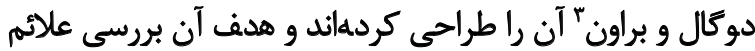

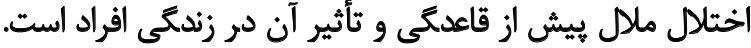

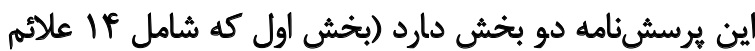

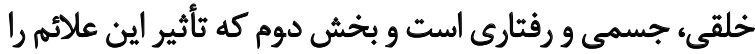

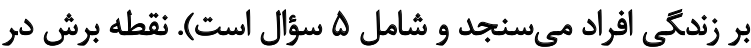

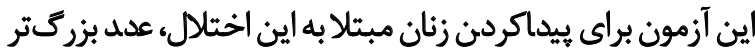

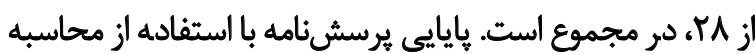
ضريب آلفاى كرونباخ بررسى شد. كرونباخ

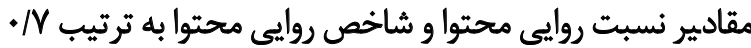

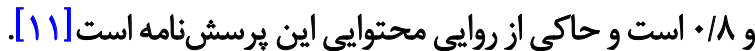

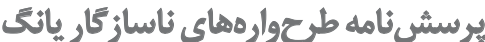

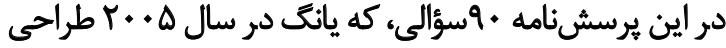

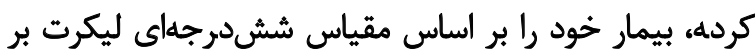

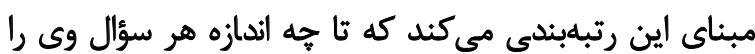

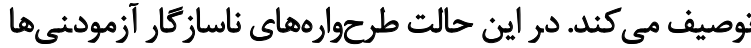

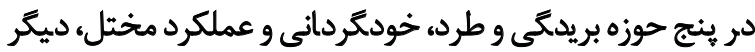

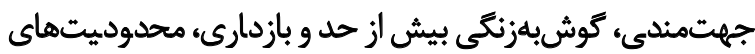

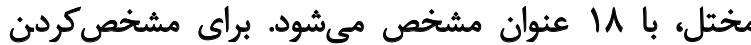

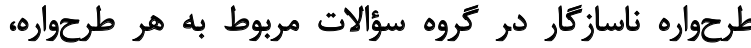

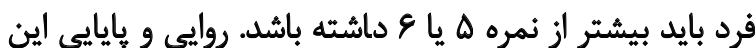
برسشنامه در هر طرحواره به صورت زير است:

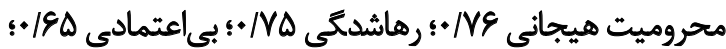

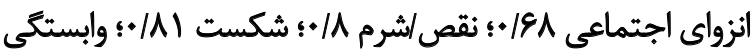

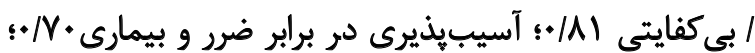

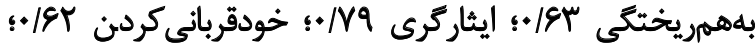

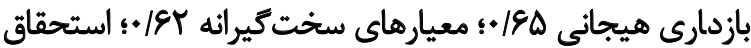

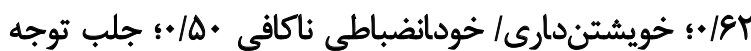

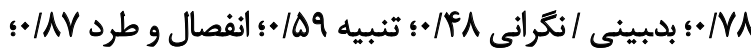

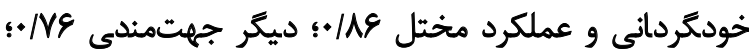

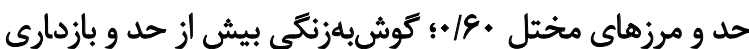

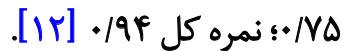

2. Premenstrual Syndrome Screening Test (PSST)

3. Steiner, Macdougall \& Brown 


\begin{tabular}{|c|c|c|c|c|}
\hline \multicolumn{3}{|c|}{ ميانكين土|نحراف معيار } & \multirow{2}{*}{ مثغير } & \multirow{2}{*}{ كروه } \\
\hline بيىكيرى & يس آزهون & بيش آزمون & & \\
\hline- & $\mid r / \Lambda+ \pm r / \Delta \Lambda$ & IfRrtr/raA & نشائههاي جسماني & \\
\hline- & IE/PVET/.PA & $18109 \pm$ Y/9Mr & اضطراب و بي خوابيى & \\
\hline- & IF/MIYH/EV. & Ir/prtr/g.1 & كاركرد اجتماعى & كنترل \\
\hline- & Ir/ute/fif & Ir/Autfiert & أفسردكى & \\
\hline- & $\triangle N N \pm V \mid F T$ & $\triangle N V E \pm N A I F$ & نمره كلى سلامت عمومى & \\
\hline $\mid f \pm m / 11 Y$ & $\mid r / \cdot v \pm r / \cdot 11$ & If/ITET/MTA & تشائههاى جسمانى & \\
\hline$I T / I T \pm M / M T$. & $11 / M^{\prime} \pm r / \cdot r \Delta$ & $\mid F / 8 \cdot \pm r / a p q$ & اضطراب و بى خوابي & \\
\hline WaY \pm H/OVD & $19 / 1 Y \pm r / V O A$ & If/gVIT/gru & كاركرد اجتماعى & آزمايش \\
\hline$N V^{\mu} \pm T / F+F$ & NA.EY/DIr & W/AVEYRATH & أفسردكى & \\
\hline$\Delta r / A \cdot \pm r / \cdot g r$ & ar/RIINIR & $\Delta \Delta / T V \pm N \cdot M$ & نمره كلى سلامت روائى & \\
\hline
\end{tabular}

مجله روانيزشكى وروانشناسى بالينى ايران

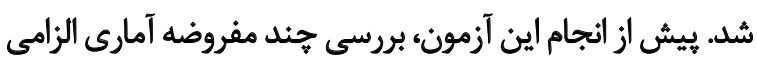

آن را تأييد كردهاند [If]

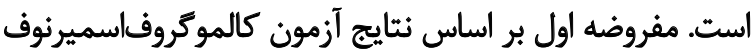
ياقتهلها

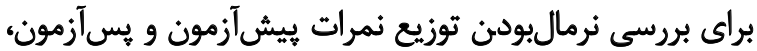

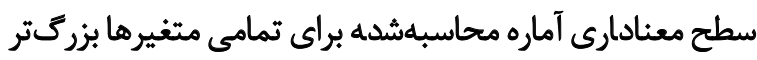

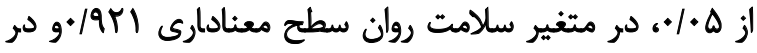

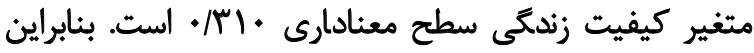

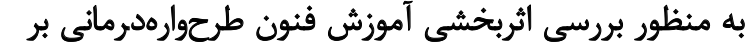

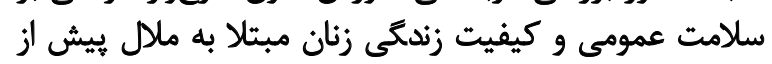

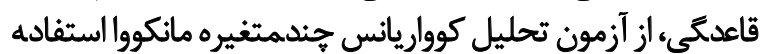

جدول ب. نمرات كيفيت زندمى در سه مرحله اندازهكيرى به تفكيك تروه آزمايش و كنترل

\begin{tabular}{|c|c|c|c|c|}
\hline \multicolumn{3}{|c|}{ ميائكين } & \multirow{2}{*}{ مثغير } & \multirow{2}{*}{ كروه } \\
\hline بيىكيرى & يس آزمون & يبش آزمون & & \\
\hline- & $|F| \backslash \pm \mid A / F Y$ & PV/WEIV/aTY & نشانههاى جسمانى & \\
\hline- & $F T / F+ \pm I r / \Delta T$ & PV/ETIT/IFA & اضطراب و بى خوابى & \\
\hline- & PNAY $\pm 19 / \Delta Q C$ & $P Q / \Delta q \pm r+1 e p q$ & كاركرد اجتماعى & كنترل \\
\hline- & $\Delta r / Q \Phi \pm I r / P A r$ & $\Delta \Delta / \Delta I \pm \mid r / M r$ & أفسردكى & \\
\hline- & $\Delta r / q r \pm r+10 \cdot q$ & PQ/TEETI/NET & نمره كلى سلامت عمومى & \\
\hline$\Delta \Delta \pm N \Delta \backslash S$ & GQ/TYEIY/RAT & $\Delta V / E V \pm I F / Q Q T$ & نشانههاى جسمانى & \\
\hline g./ArtQ/Trr & $\Delta V / V A \pm 1+/ 1 q$ & $|\Delta \pm 1| / 91 \mid$ & اضطراب و بى خوابى & \\
\hline$\Delta \& / 11 \pm 1 \Delta / \Delta \Lambda$ & $\Delta r / F f \pm 1 \Delta /+g r$ & FNAq IV/Fqq & كاركرد اجتماعى & آزمايش \\
\hline$g F / \Delta \wedge \pm q / \Lambda \Delta q$ & $g+ \pm \mid r / A F I$ & $\Delta F / \Delta A \pm I \Delta / M \Delta$ & اقسردگى & \\
\hline 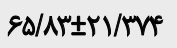 & SAINNTE. & $\Delta Q / \Delta r \pm \mid \Delta / \Delta V^{e}$ & نمره كلى سلامت روائي & \\
\hline
\end{tabular}


علائم افسردكى دربركيرنده مواردى است كه با احساس ناميدى،

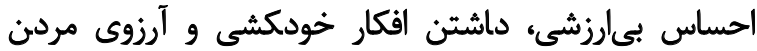

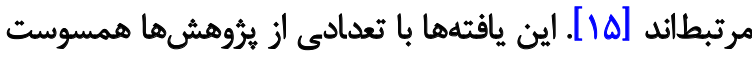

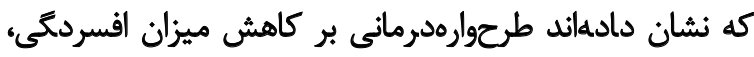

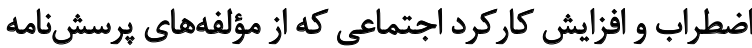

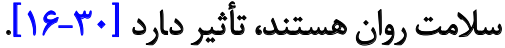

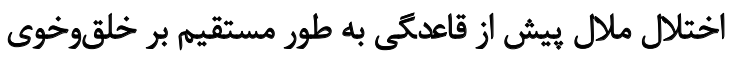

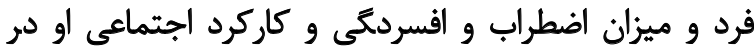

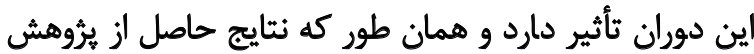

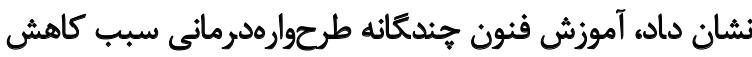

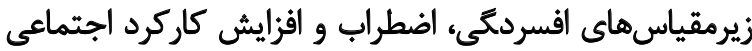

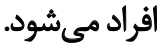

مرحله دوم: آموزش فنون طرحوارددرمانى بر كيفيت زندمَى

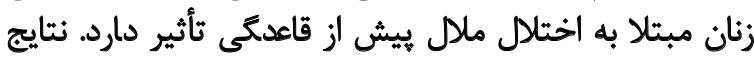

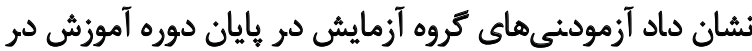

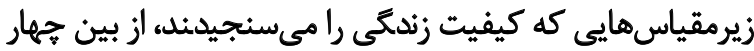

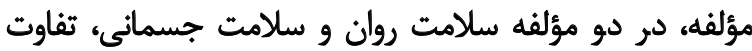

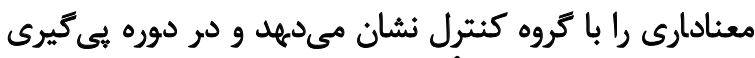

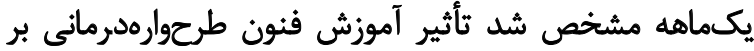

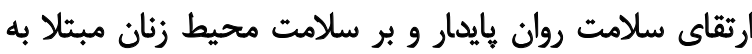
اختلال ملال ييش از قاعدكى، مؤثر است.

طبق تعاريف، حوزه سلامت جسمى شامل فعاليتهاى

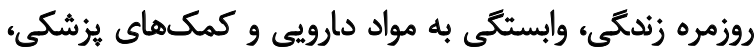

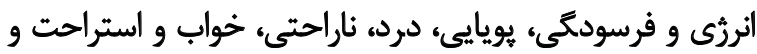

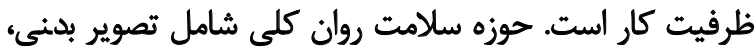

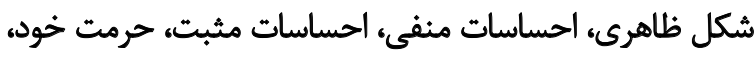

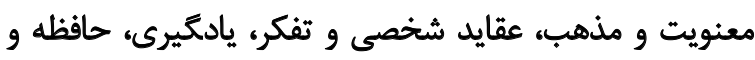

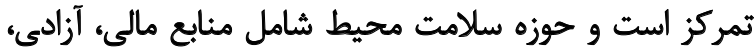

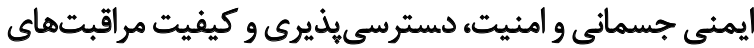

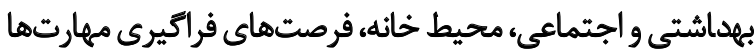

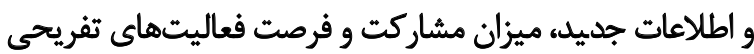

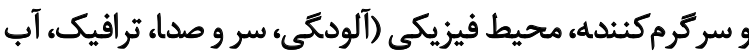

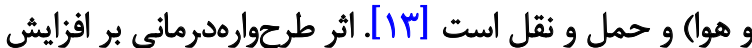

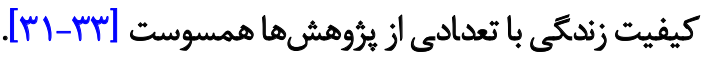
اختلال ملال بيش از قاعدگى به دليل تغييرات مخرب و ايجاد

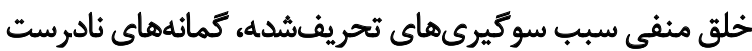

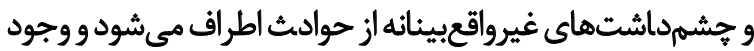

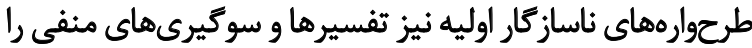

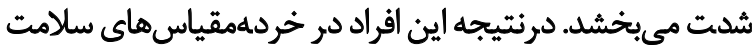

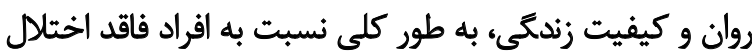

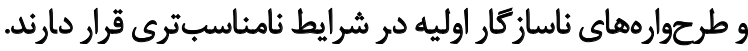
بيشتر باورهاى ناكارآمدى كه در افراد كروه آزمايش مشاهده
فرض نرمالبودن توزيع نمرات يذيرفته مىشود. يكى ديكر از مفروضات اجراي آزمون تحليل كوواريانس

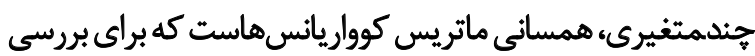

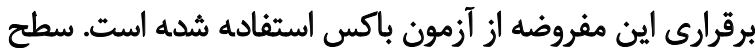

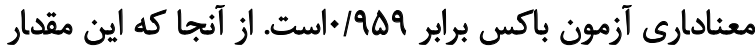

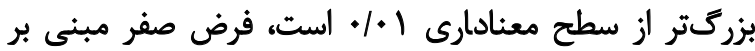
همسانى ماتريس كوواريانس ها برقرار است. يكى ديكر از مفروضات اجراى آزمون تحليل كوواريانس

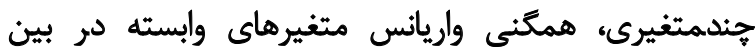

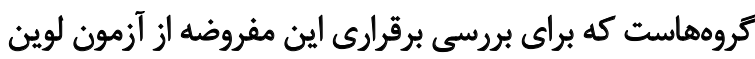

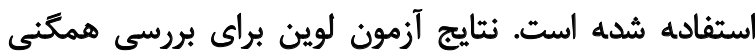

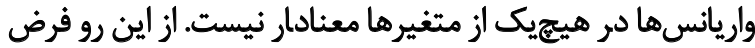

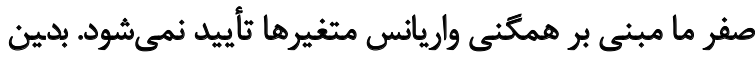

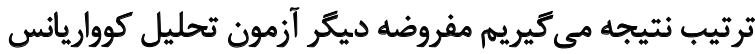

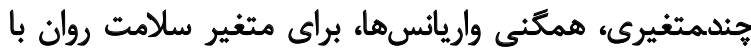

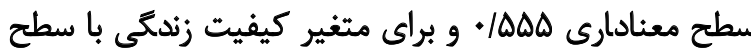

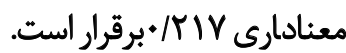

با توجه به جدول شماره ז، ميانگين نمرات تروه كنترل در

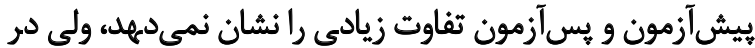

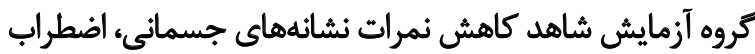

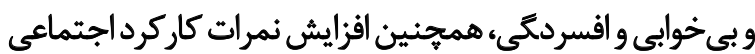

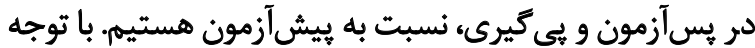

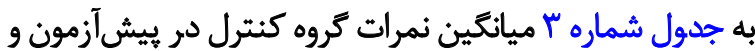

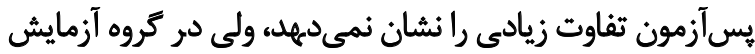

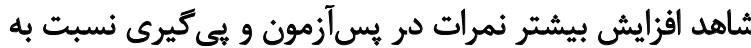
بيشآزمون هستيهم

بحث يافتههاى اين برؤهش در دو مرحله تبيين مىشود. مرحله

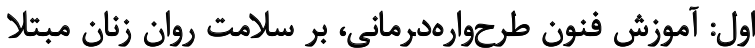

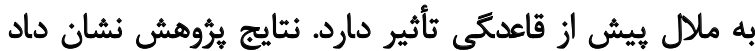

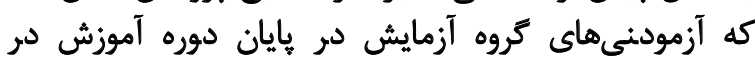

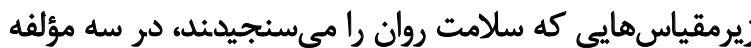

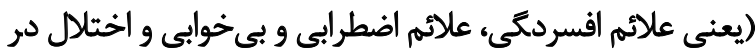

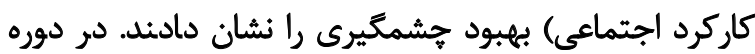

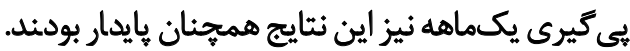

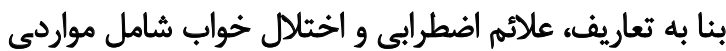

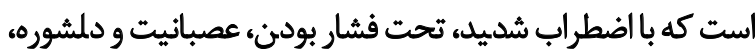

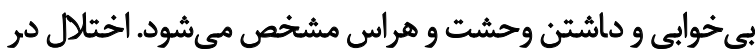

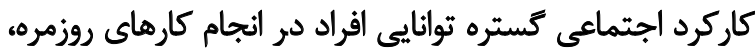

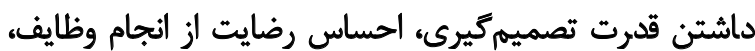

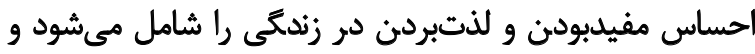


تكنيكهاي شناختى به مراجعان كمك مي كند ثااز طرحوارهها

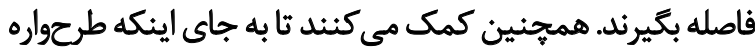

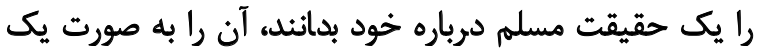

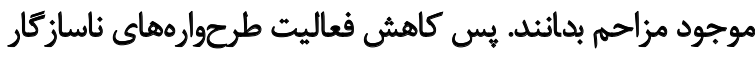

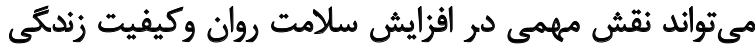

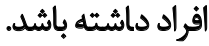

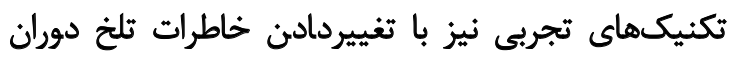

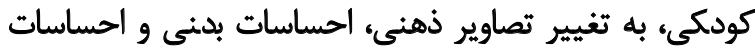

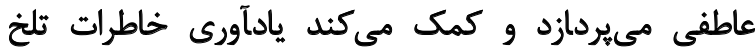

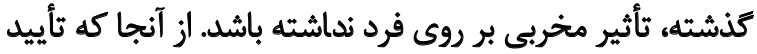

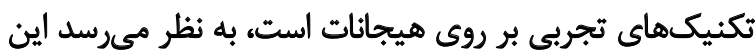

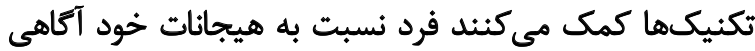

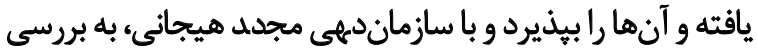

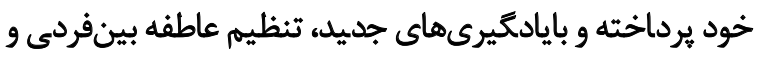

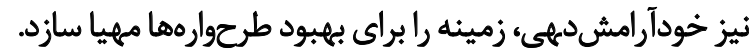

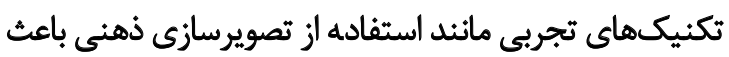

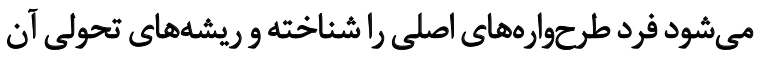

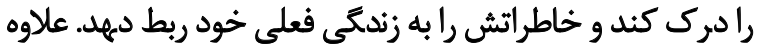

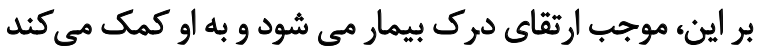

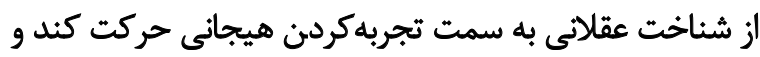

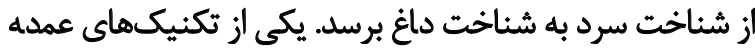

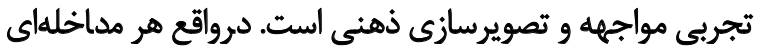

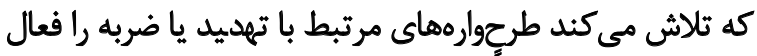

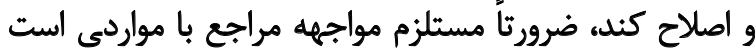

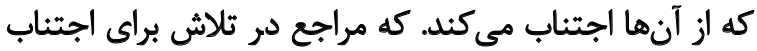

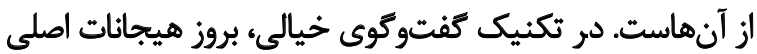

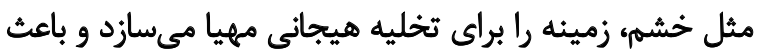

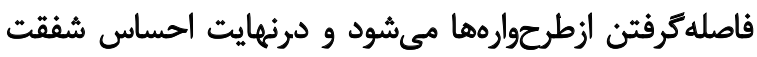

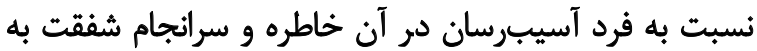
كودك آسيبديده را باعث ميشود آسيب.

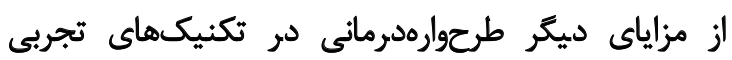

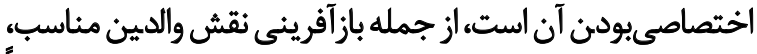

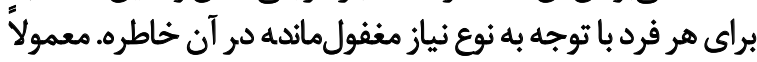

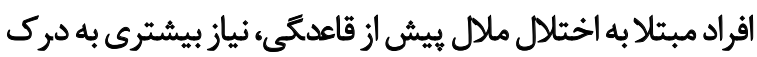

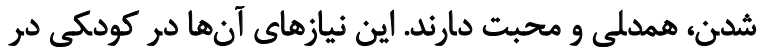

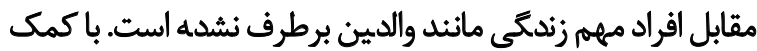

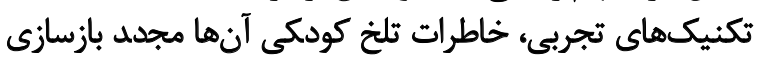

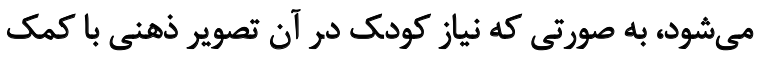

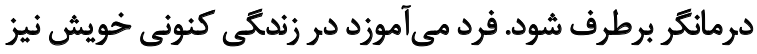

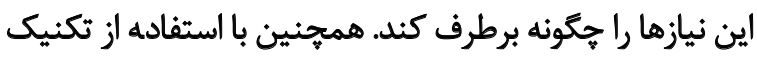

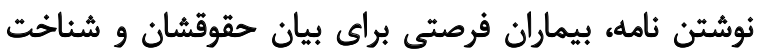

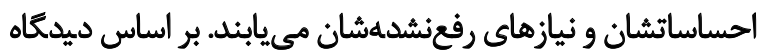
يانك و همكاران هدف طرحوارهدمانى كمك به به رفع نيازهاى

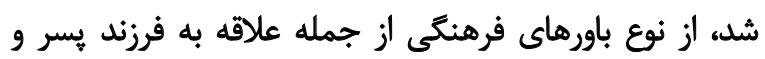

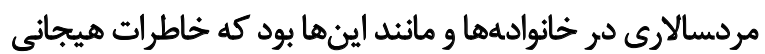

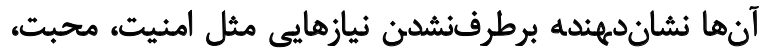

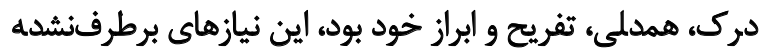

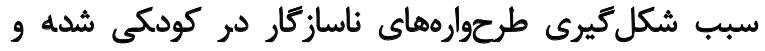

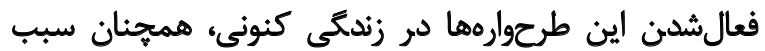
رفعنشن اين نيازها مىشود.

آموزش فنون شناختى در طرحوارهدرمانى سبب شد آزمودنىها

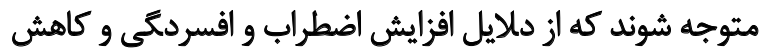

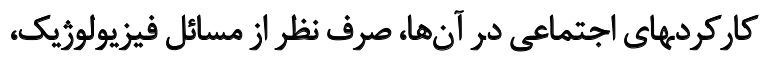

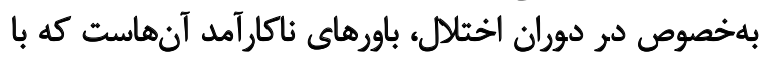

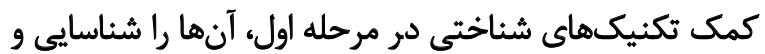

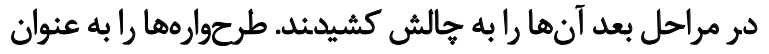

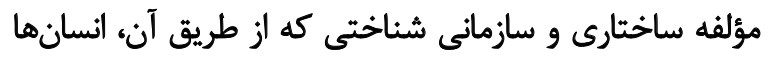

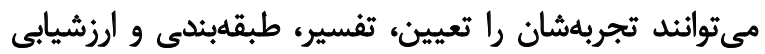

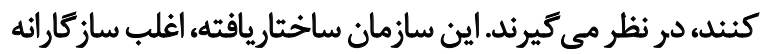

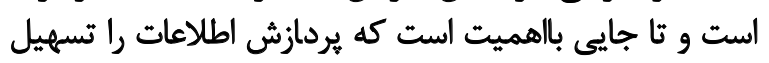

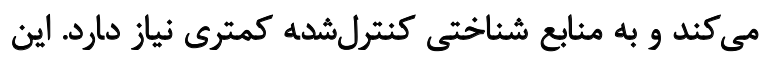

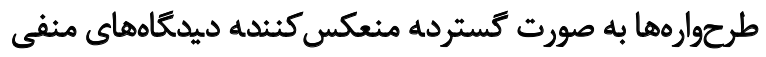
مطلق فرد نسبت به خود، دنيا و آينده است

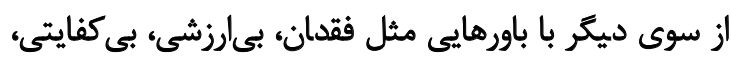

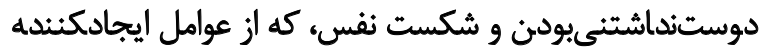

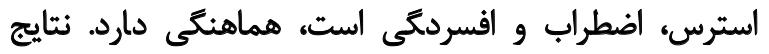

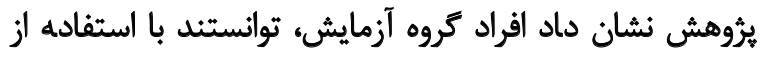

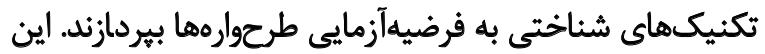

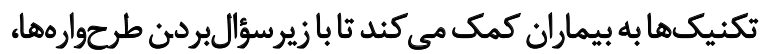

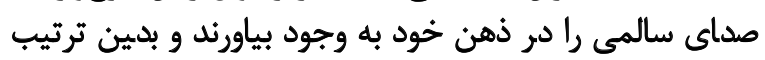
ذهنيت سالم خود را توانمند سازند.

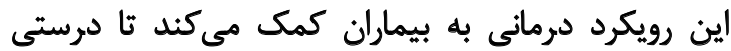

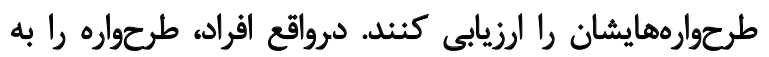

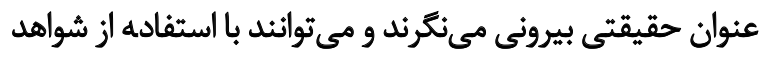

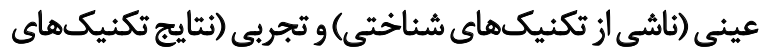

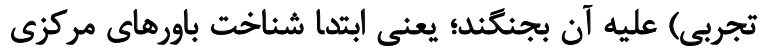

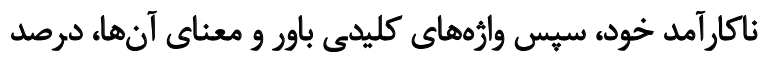

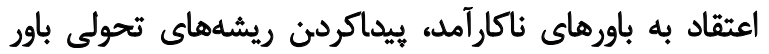

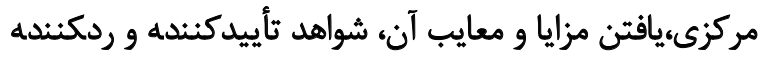

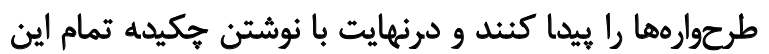

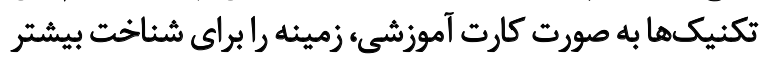

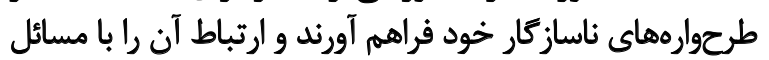

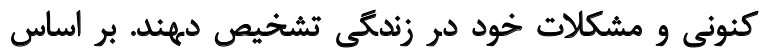

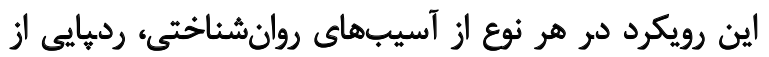

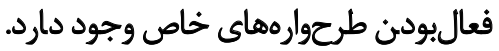


يكي ديكر از دلايل ائربخشى طرحوارهدرماني استفاده از

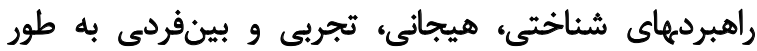

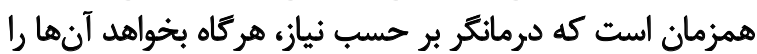

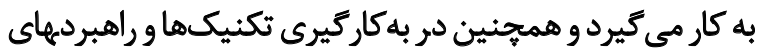

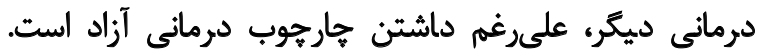

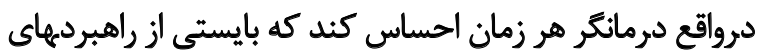

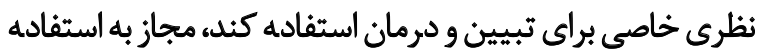

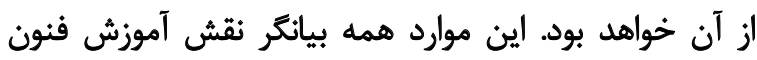

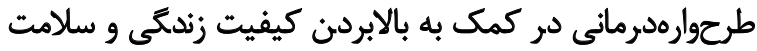

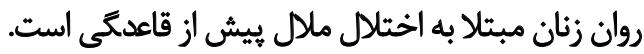

\section{نتيجليَّى}

طبق نتايج آمارى و مشاهدات بالينى بهدستآمده از اين

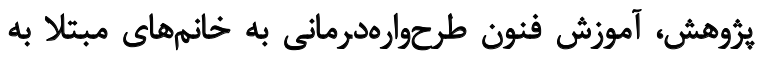

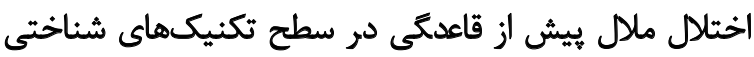

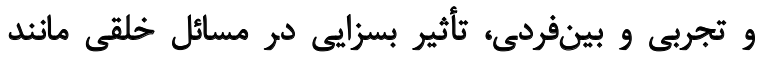

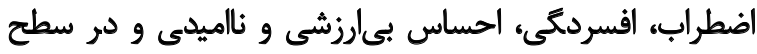

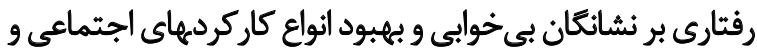

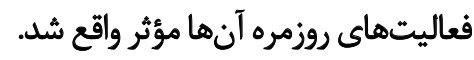

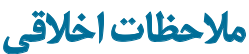

$$
\text { يبيروى از اصول اخغلاق بورهش }
$$

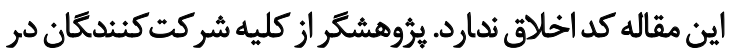

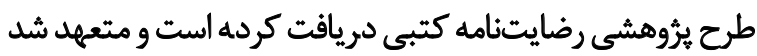

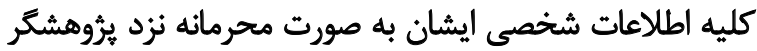
محفوظ مى ماند.

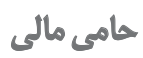

اين مقاله بركرفته از قاياننامه كارشناسيارشد نويسندها

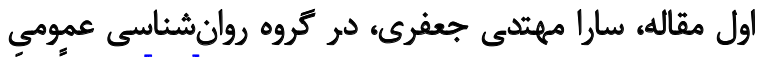

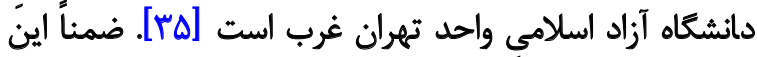

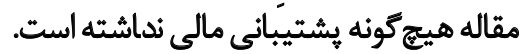

$$
\text { مشاركت ثويسند متَان }
$$

تمامى بخشهاي اين مقاله را نويسنده اصلى (سارا مهتدى جعفرى) انجام داده است.

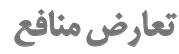

بنابر اظهار نويسندكان، اين مقاله تعارض منافع ندارد.

$$
\text { هيجانى بيمار است. }
$$

زمانى كه در طى فرايند درمان اين نيازهاى هيجانى تاحدودىى ئري

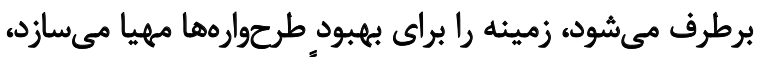

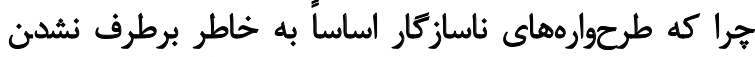

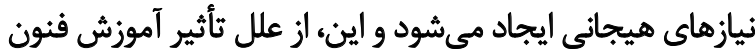

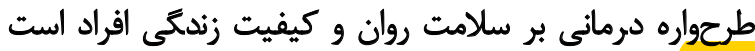

[rF]

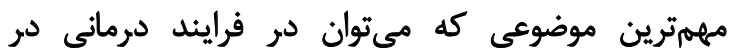

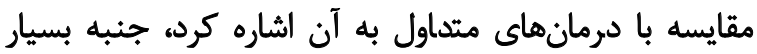

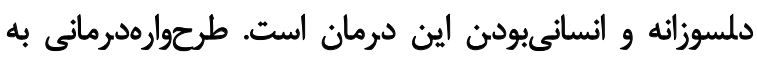

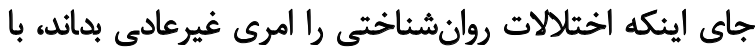

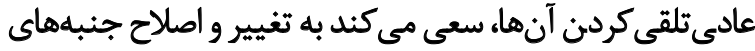

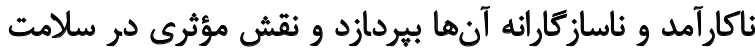

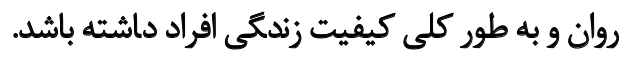

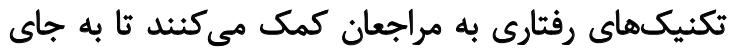

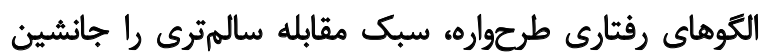

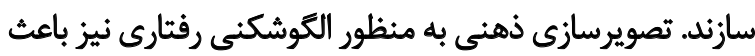

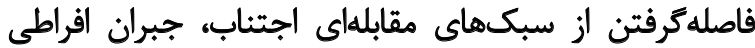

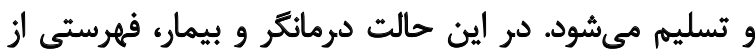

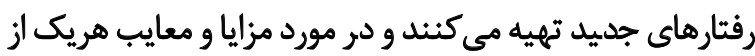

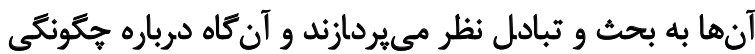

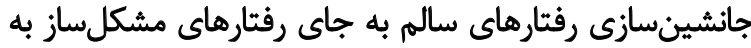

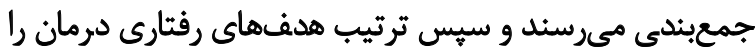

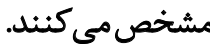

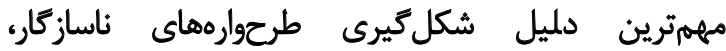

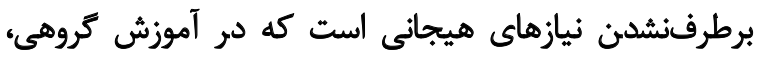

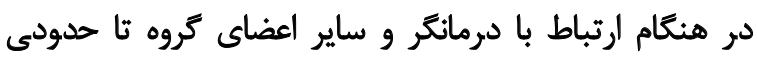

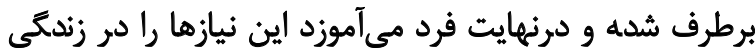

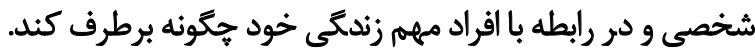

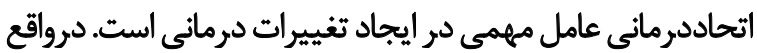

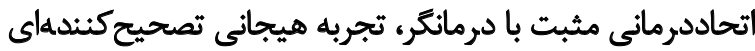

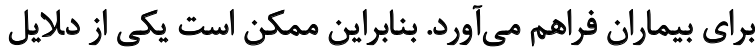

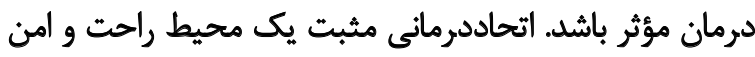

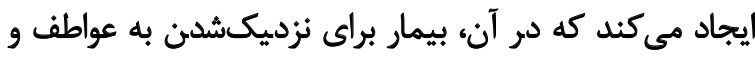

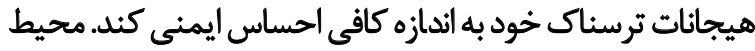

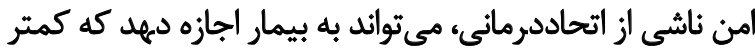

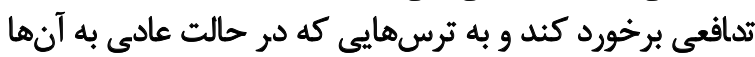

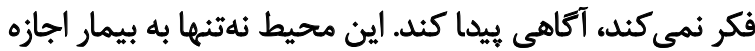

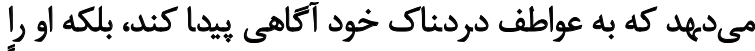

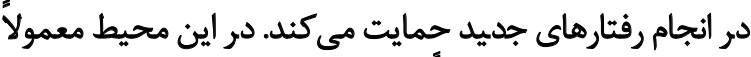

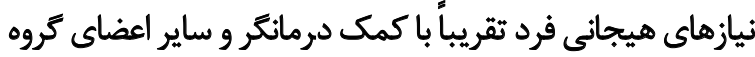
برطرف شده و نوعى بازوالدينى انجام مى كيردي 


\section{References}

[1] Baghdassarians A, Bagheri Karimi A. Applied treatment of PMS. Tehran: The Voice of Light; 2014

[2] Navabinejad S, Dadvandi M. [Step by step guide to the treatment of mental and physical problems in menstruation (Persian)] Tehran: Perkas; 2013.

[3] Armand A, Talaei A. Effect of cognitive-behavioral stress management training on reducing psychological problems and symptoms of premenstrual dysfunction in affected women (Persian)]. Journal of Women. 2012; 15(21):31-24.

[4] Puriyamanesh L, Shahrjerdi SH, Karimizadeh Ardakani M, Ansari MA. [Relationship Between Abnormalities Between Quality of Life and Mental Health of Female Students of Faraghani University of Arak (Persian)]. Journal of Shahrekord University of Medical Sciences. 2017; 19(2):41-8.

[5] Theofilou, P. Quality of life: Definition and measurement. Europe's Journal of Psychology. 2013; 9(1):150-62. [DOI:10. 5964/ ejop. v9i1. 337]

[6] Akbari E, Mahmoud Aliloo M, Khanjani Z, Poursharifi H, Fahimi S, Amiri Pichakolaei A, et al. [Early maladaptive schemas and parenting styles of students who have failed in love with and without the clinical syndrome (Persian)]. Iranian Journal of Psychiatry \& Clinical Psychology. 2012; 18(3):184-94.

[7] Young J, Colosco J, Vishar M. Health Plan. A Guide for Clinical Practitioners. [H Hamidpour, Z Andouz, Persian Trans.]. Tehran: Arjmand; 2012.

[8] Sayadi G, Golmohammadian M, Rashidi A. [The effectiveness of schema therapy on the undermining of teenager girls of divorce families (Persian)]. Journal of Clinical Psychology Researches and Advice of Ferdowsi University of Mashhad. 2017; 7(1):1-8

[9] Ramazanpour F, Bahri N, Fathi Najafi T. [Status and severity of premenstrual syndrome and its relationship with the personal and social characteristics of Gonabad students (Persian)]. Iranian Journal of Obstetrics and Gynecology and Infertility. 2015; 18(169):21-8.

[10] Hallgin R, Whitburne P, Cross S, Seyyed Mohammadi Y. [Psychological pathology, clinical perspectives on psychiatric disorders (Persian)]. Teharn: Ravand; 2013.

[11] Siahbazi SH, Hariri F, Montazeri A, Moghadam L. [Standardization of PSST Screening Questionnaire (Persian)]. Iranian Translations and Psychometrics. 2011; 10(4):422-7.

[12] Young JV, Klosko J, Wieshaar M. Schema therapy: Apractitioner's guide. New York: Guilford Press; 2003. [DOI:10. 1007/ s11630-003-0064-2

[13] Taghavi SMR. Validity and validity of General Health Questionnaire (Persian)]. Journal of Psychology; 5(4):381-98.

[14] Nejat SN. [Quality of life and measurement (Persian)]. Iranian Journal of Epidemiology. 2008; 4(2):5-7.

[15] Aminpour H, Zare H. Application of psychological tests. Tehran: Payame Noor University; 1395

[16] Malekpour Dehkordi E. [The effect of schematic therapy on depression depression, ineffective attitude and negative self-concept in students (Persian)] [MSc. thesis]. Payame Noor University, Tehran, Isfahan University of Medical Sciences.
[17] Zerehpoush A, Neshatdoust HT, Asgari K, Abedi MR, Sadeghi Otejia AH. [The effectiveness of schema therapy on chronic depression in students (Persian)]. Journal of Behavioral Sciences Research. 2012; 10(4):285-91.

[18] Tabatabaeibarzaki S, Sohrabi S. [The effectiveness of training schema therapy's experiential techniques on the depressed persons' schemas (Persian)]. Counseling Culture and Psychotherapy. 2012; 3(11):75-86.

[19] Majid Saffarinia, Hossein Zare, Jahangir Karami, Zahra Solgi. [The efficacy and continuing impact of group schema therapy in treating students' social anxiety disorder (Persian)]. Pajoohande. 2014; 19(4):211-8.

[20] Amirahmadi M, Razeghi N, Aghaee H. [Comparing the effectiveness of neurofeedback and schematherapy on symptoms of depression in depressed women in Tehran (Persian)]. Neuropsychology. 2016; 1(3):97-114

[21] Asefi R, Alipour A, Safari Nia M. [Comparison of the effectiveness of therapeutic spirituality and schema therapy on depression of students (Persian)]. Ravanshenasi. 2016; 9(2):27-42.

[22] Hemmati Sabet Vahid, Rouhani Shahrakestani N, Hemmati Sabet A, Panah Mohammad A. [The effectiveness of schema therapy in reducing aggression and social anxiety in adolescents of Hamedan City aged 17 to 18 years (Persian)]. Shenakht Journal of Psychology and Psychiatry. 2016; 3(2):82-93.

[23] Homayoun M. [The effectiveness of schema therapy on the treatment of general anxiety disorder (Persian)] [MSc. Thesis] Islamic Azad University of Marvdasht; 2017.

[24] Mozamzadeh T, Gholamrezai S, Rezaei F. [The effect of schema therapy on severity of depression and suicidal thoughts in patients with resistant to depression (Persian)]. Armaghan-e Danesh. 2018; 23(2):253-66.

[25] Hosseini Fard SM. [Depression, despair, initial maladaptive schemes, increased self-esteem and self-esteem (Persian)] [PhD. dissertation]. Tehran: University of Social Welfare and Rehabilitation Sciences; 2018

[26] Jovev M, Jackson HJ. Early maladaptive schemas in personality disordered individuals. Journal of Personality Disorders. 2004 18(5):467-78. [DOI:10. 1521/pedi. 18. 5. 467. 51325] [PMID]

[27] Lumley MN, Harkness KL. Specificity in the relations among childhood adversity, early maladaptive schemas, and symptom profiles in adolescent depression. Cognitive Therapy and Research. 2007; 31(5):639-57. [DOI:10. 1007/ s10608-006-9100-3]

[28] Hawke LD. Schema theory and schema therapy in mood and anxiety disorders: A review. Journal of Cognitive Psychotherapy. 2011; 25(4):257-79. [DOI:10. 1891/0889-8391. 25. 4. 257]

[29] Malogiannis IA, Arntz A, Spyropoulou A, Tsartsara E, Aggeli A, Karveli S, Vlavianou M, Pehlivanidis A, Papadimitriou GN Zervas I. Schema therapy for patients with chronic depression: A single case series study. Journal of Behavior Therapy and Experimental Psychiatry. 2014; 45(3):319-29. [DOI:10. 1016/j. jbtep. 2014 02. 003] [PMID]

[30] Carter JD, McIntosh VV, Jordan J, Porter RJ, Douglas K, Frampton CM, et al. Patient predictors of response to cognitive behaviour therapy and schema therapy for depression. Australian \& New Zealand Journal of Psychiatry. 2018; 52(9):887-97. [DOI:10 1177/0004867417750756] [PMID] 
[31] Dickhaut V, Arntz A. Combined group and individual schema therapy for borderline personality disorder: A pilot study. Journal of Behavior Therapy and Experimental Psychiatry. 2014 45(2):242-51. [DOI:10. 1016/j. jbtep. 2013. 11. 004]

[32] Mohammad Nezhady B, Rabiei M. The effect of schema therapy on quality of life and psychological well-being in divorced women. Journal of Police Medicine. 2015; 4(3):179-90.

[33] Rahimaghaee F, Hatamipour K, Ashoori J. [The effect of group schema therapy on symptoms of depression and quality of life among nurses (Persian)]. Journal of Nursing Education. 2017; 6(23):17-23. [DOI:10. 21859/jne-06033]

[34] Kellogg SH, Young JE. [Schema therapy for borderline personality disorder (Persian)]. Journal of Clinical Psychology. 2006; 62(4):445-58. [DOI:10. 1002/jclp. 20240] [PMID]

[35] Mohtadi Jafari S, Ashayeri H, Banisi P. [The effectiveness of teaching schema therapy techniques in mental health and quality of life of women with premenstrual dysphoric disorder (Persian)] [MSc. thesis]. Tehran: Islamic Azad University; 2018. 
This Page Intentionally Left Blank 